\title{
THE INTRINSIC MAGNETIC FIELD ORIENTATION \\ IN NGC4631: \\ DYNAMO OR OUTFLOW ?
}

\author{
GÖTZ GOLLA \\ Max-Planck-Institut für Radioastronomie, Auf dem Hügel 69, D-5300 Bonn 1, Germany \\ and \\ EDSKO HUMMEL \\ Royal Observatory, Blackford Hill, Edinburgh, EH9 $3 H J$, Scotland
}

\begin{abstract}
The interacting galaxy NGC4631 is unique among edge-on galaxies in that it shows a prominent synchrotron radio halo. We derive the intrinsic magnetic field structure in this halo. The field lines are mainly radially outgoing from the central region. The possible mechanisms generating such magnetic fields in the halo, i.e. outflows and dynamo processes, are discussed.
\end{abstract}

\section{Introduction}

NGC 4631 is a late-type edge-on spiral galaxy showing a wealth of HII regions and a chaotic distribution of dust on optical pictures. The galaxy is interacting with two neighbours, NGC4627 and NGC4656. The eastern part of NGC4631 has been distorted during the passage of NGC4656 about $3 \times 10^{8}$ years ago (Combes 1978).

The central region of the galaxy exhibits a modest starburst (Golla 1992). Such central starbursts are quite common among interacting galaxies and are believed to be triggered by the interaction.

NGC4631 is well-known for its spectacular radio halo. Synchrotron emission is found more than $8 \mathrm{kpc}$ above the plane, implying the presence of cosmic rays and magnetic fields at such heights. Hummel et al. $(1988,1991)$ investigated the polarized radio emiss ion of the halo and found high degrees of polarization (up to $30 \%$ ) and thus a high degree of order of the magnetic field.

The orientation of the magnetic field can in principle be derived from measurements of the radio polarization angle. However, these angles are usually altered by Faraday rotation when the radiation transverses a magnetized plasma with a magnetic field component parallel to the line of sight. Hummel et al. (1991) proposed that the electron density in the halo of NGC4631 is low enough that Faraday rotation can be neglected. Based on this assumption they found the magnetic field orientation to be predominantly perpendicular to the plane of NGC4631.

\section{The Intrinsic Magnetic Field Orientation in NGC4631}

We undertook a multifrequency study of the polarized emission of NGC4631 with the VeryLargeArray at $3.5 \mathrm{~cm}$ and $6 \mathrm{~cm}$ wavelength (Golla \& Hummel 1992) and with the Effelsberg $100 \mathrm{~m}$ telescope at $2.8 \mathrm{~cm}$ wavelength (Golla 1992). Additionally $20 \mathrm{~cm}$ VLA data were used from Hummel et al. $(1988,1991)$. The aim was to derive the rotation measures utilizing the wavelength dependence $\left(\propto \lambda^{2}\right)$ of Faraday rotation and to determine the intrinsic field orientations in the halo. 
Fig. 1. Intrinsic magnetic field orientation of NGC4631, 84" resolution, contour plot of the total intensity at $\lambda 2.8 \mathrm{~cm}$, overlayed on an optical $R$ band image

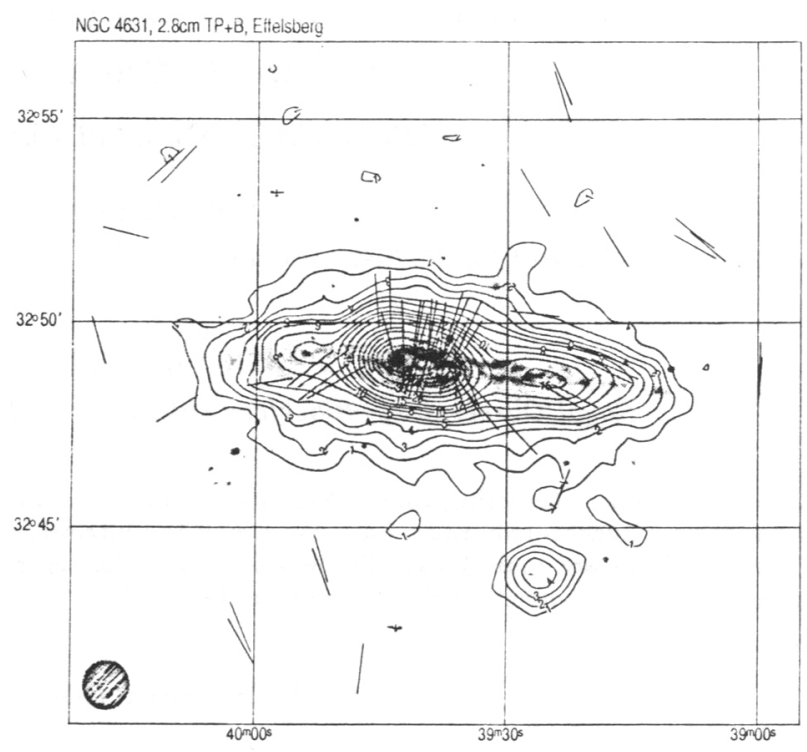

The low resolution Effelsberg data at $2.8 \mathrm{~cm}$ exhibit already such a low Faraday rotation that a correction is not necessary. Figure 2 shows the magnetic field orientation derived from these data together with contours of total intensity at $2.8 \mathrm{~cm}$. Clearly the field lines are perpendicular to the plane of NGC4631 above its central region. The assumption of Hummel et al. of negligible Faraday rotation is therefore mostly correct.

The intrinsic magnetic field orientation of the VLA data was derived combining two wavelength pairs, $20 \& 6 \mathrm{~cm}$ and $6 \& 3.5 \mathrm{~cm}$. Two maps of the magnetic field orientation were obtained in this way with two different angular resolutions: 40" (using the $20 \& 6 \mathrm{~cm}$ VLA data) and 12" (using the $6 \& 3.5 \mathrm{~cm}$ VLA data).

Figure 3 reveals the field direction with 40 " $(1.5 \mathrm{kpc})$ resolution together with contours of the total intensity at $6 \mathrm{~cm}$ wavelength. The field seems to be radially outgoing from the central region and perhaps from some parts of the eastern disk. In Figure 3 the most detailed (12", 440pc resolution) image of the field structure near the central region is given. Most of the field lines seem to emerge from the central region, where a triple source can be identified in total intensity. Northeast of the estern triple source the B-field lines correlate well with a radio spur, which is found to be highly polarized.

\section{The Origin of the Radio Halo and its Magnetic Field Structure}

The radio halo of NGC4631 is not just a diffuse sphere but shows considerable structure in the form of 'spurs'. These spurs can be discerned in the total intensity maps in various sizes and lengths, beginning from a few $100 \mathrm{pc}$ up to almost $10 \mathrm{kpc}$. The footpoints of the spurs are often connected to star forming regions in the disk. In all cases where magnetic field lines were determined they seem to run parallel to the spurs (cf. Golla \& Hummel 1992). 


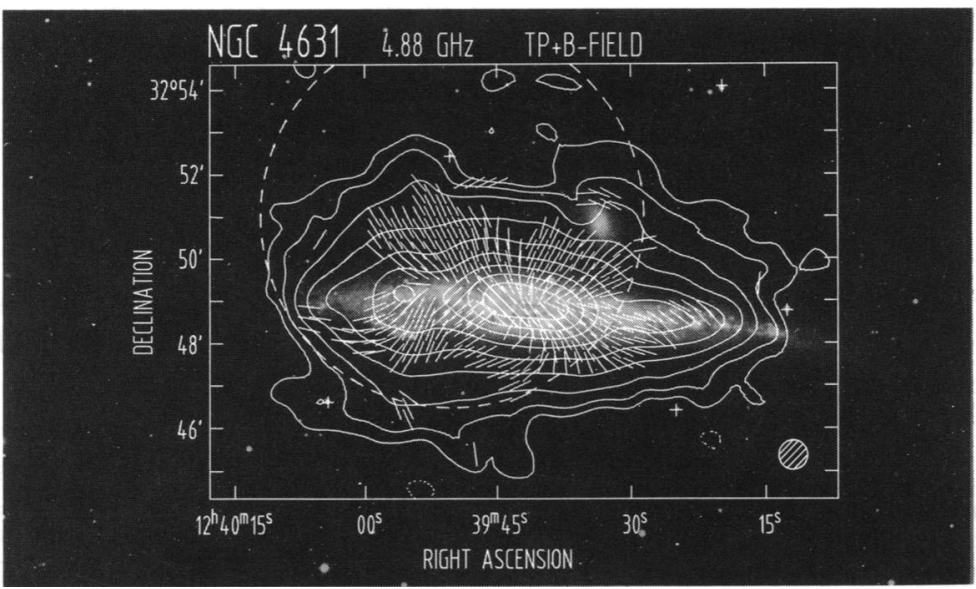

Fig. 2. Intrinsic magnetic field orientation, 40 " resolution, contour plot of the total intensity at $\lambda 6 \mathrm{~cm}$, overlayed on an optical image, circle denotes the diameter of the primary beam

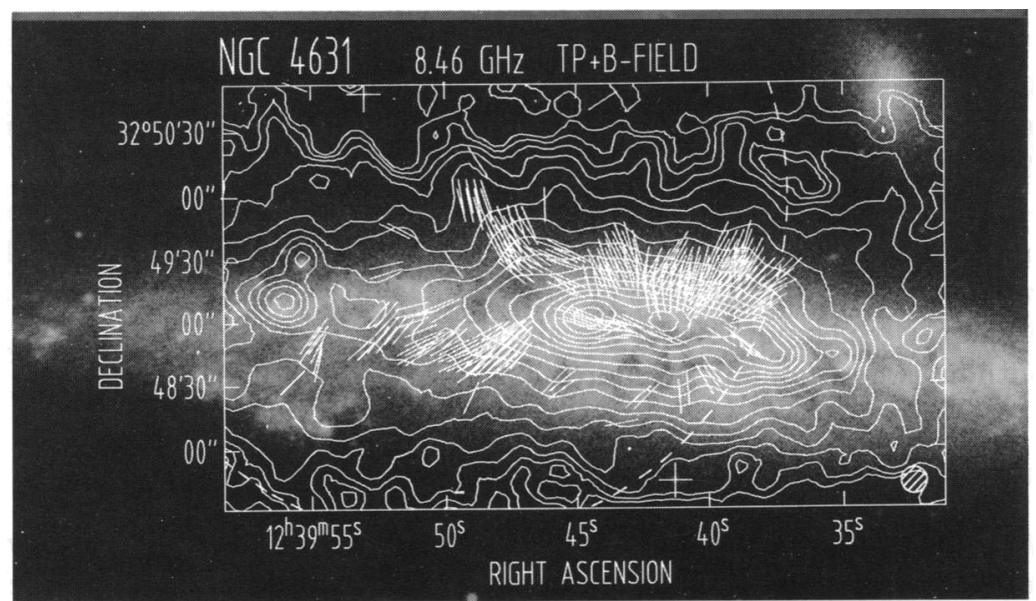

Fig. 3. Intrinsic magnetic field orientation, 12 " resolution, contour plot of the total intensity at $\lambda 3.5 \mathrm{~cm}$, overlayed on an optical image, circle denotes the diameter of the primary beam

It is generally accepted that cosmic rays are produced in supernova remnants . Therefore it is rather straightforward to propose that the cosmic rays in the radio halo of NGC4631 originate from its disk and propagate along the magnetic field lines into the halo. Then the question arises how the magnetic field structure in the halo is generated. The answer is an essential part of any explanation of the origin of the extraordinary radio halo itself.

Theoretically, the origin of the B-field in the halo can be either an outflow from 
the plane (the B-field is transported out of the disk together with the gas it is frozen into) or a large-scale dynamo. Both processes may exist concurrently, the dynamo field eventually supporting the outflow out of the central region or the outflow distorting the dynamo field.

\subsection{An Outflow into the Halo}

There are many indications for outflow phenomena in the disk-halo interface of NGC4631. HI observations (Weliachew et al. 1978) give evidence that gas was pulled out of the disk during the encounter of NGC4631 with NGC4656.

The high star formation rate in the central region makes it probable that correlated supernovae explosions produce superbubbles of hot gas and cosmic rays. When the superbubbles reach a certain size they release their contents into the halo. Depending on the density of the bubbles in the plane there will be local outflows ("chimneys") or an overall wind (Norman \& Ikeuchi 1989). Evidence for such outflows was found by Rand et al. (1992) who detected vertical H $\alpha$ features ('worms') above the central region. These worms correlate with the observed small scale radio spurs (Golla 1992).

Purely cosmic-ray driven winds may also exist. Breitschwerdt et al. (1991) modeled such galactic winds. They found that outflow velocities of $50 \mathrm{~km} / \mathrm{sec}$ in the first $\sim 10 \mathrm{kpc}$ and terminal velocities of more than $\sim 150 \mathrm{~km} / \mathrm{sec}$ may be reached. Although there is no direct measurement of an outflow velocity indirect evidence has been found in form of spectral index variations (Hummel \& Dettmar 1990, Golla 1992).

NGC4631 has a rather low mass, as indicated by the low rotation velocity of $\approx 140 \mathrm{~km} / \mathrm{s}$ (Golla 1992). Additionally one the neighbouring galaxies, NGC4627, is probably situated just north of the plane of NGC4631. Therefore the gravitational potential against which outflowing gas and CRs have to move is comparably weak and an outflow is additionally promoted.

\subsection{Large-Scale Dynamo Activity}

A variety of dynamo scenarios and models has been presented in the last few years. For example Elstner et al. (1992) presented a disk dynamo model in which they considered turbulent motions with non-vanishing helicity ( $\alpha$-effect) and differential rotation of the disk ( $\omega$-effect). In NGC4631 star formation and thus enhanced turbulence is concentrated in the inner, rigidly rotating part of the disk. This means that the $\omega$ effect cannot be utilized for significant dynamo action. An $\alpha^{2}$-dynamo instead of an $\alpha \omega$-dynamo must be involved. Elstner et al. modeled such an $\alpha^{2}-$ dynamo based on the rotation curve of NGC4631 and found that a dipolar magnetic field structure reaching out into the disk-halo interface can be produced. However, the observed magnetic field is more conical and the observed rotation measure distribution does not agree with a pure dipolar field configuration.

Brandenburg et al. (1992a) modeled combined disk and halo dynamos, utilizing differential rotation in disk and halo. They state that the magnetic field in the halo is oscillatory and that due to the large size of the dynamo volume, the period of the 
oscillations is comparable to the Hubble time. This means that any existing halo dynamo will not be in steady state and a variety of magnetic field morphologies should occur in galaxies. Due to the large growth time a halo dynamo in NGC4631 could not be caused by interaction and the resulting star formation activity $\approx 3 \times 10^{8}$ years ago. If the radio halo of NGC4631 is caused by such a halo dynamo one should be able to find at least some galaxies with similarly prominent radio halos and magnetic field structures. Hummel et al. (1991b) observed a sample of 181 edge-on galaxies and did not succeed in finding galaxies with radio halos comparable to the one of NGC4631.

\section{Discussion}

Based on the observational data available it is almost certain that an outflow or wind of gas and cosmic rays from the disk into the halo of NGC4631 is taking place. This wind can probably account for the observed magnetic field configuration in the halo and thus also for the radio halo itself.

The existing observations and dynamo models do not allow to evaluate the relevance of a dynamo as the source of or as a contributor to the halo magnetic field of NGC4631. When models consider the physical processes only very crudely whereas the observed $d$ ata are a reflection of a multitude of detailed processes, the direct comparison of model results and observations is of limited value. It is then almost impossible to verify or falsify the existence of a dynamo.

Dynamo models for NGC4631 have until now not considered the presence of a galactic wind. This is a must for future models, since a wind may severely modify the dynamo. Either it can advect the magnetic field before it can be amplified or it can under certain circumstances even promote a dynamo. The influence of velocity fields other than the usual differential rotation on dynamos is just at the beginning of being investigated. First work has been done by Spencer \& Cram (1992) and by Brandenburg et al. (1992b), who included a wind-like velocity field in the halo into their numerical dynamo code.

\section{References}

Brandenburg A., Donner K.J., Moss D., Shukurow A., Sokoloff D.D., Tuominen I., 1992, A\&A 259,453

Brandenburg A., Donner K.J., Moss D., Shukurow A., Sokoloff D.D., Tuominen I., 1992b, A\&A (submitted)

Breitschwerdt D., McKenzie J.F., Völk H.J., 1991, A\&A 245, 79

Combes F., 1978, A\&A 65, 47

Elstner D., Meinel R., Beck R., 1992, A\&AS 94, 587

Golla G., 1992, PhD thesis, University of Bonn

Golla G., Hummel E., 1992, A\&A (submitted)

Hummel E., Lesch H., Wielebinski R., Schlickeiser R., 1988, A\&A 197, L29

Hummel E., Beck R., Dahlem M., 1991, A\&A 248, 23

Hummel E., Beck R., Dettmar R.-J., 1991b, A \& A 248, 23

Hummel E., Dettmar R.-J., 1990, A\&A 236, 33

Norman C.A., Ikeuchi S., 1989, ApJ 345, 372

Rand J. R., Kulkarni S. R., Hester, S.J., 1992, ApJ 396, 97

Spencer S.J., Cram L.E., 1992, A\&A 400, 484

Weliachew L., Sancisi R., Guélin M., 1978, A\&A 65, 37 